\title{
Kinetic Control of Perovskite Thin-Film Morphology and Application in Printable Light-Emitting Diodes
}

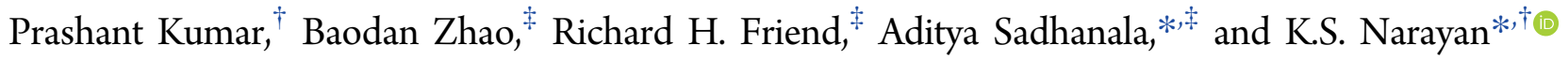 \\ $\dagger$ Jawaharlal Nehru Centre for Advanced Scientific Research, Bangalore 560064, India \\ ${ }^{\ddagger}$ Cavendish Laboratory, University of Cambridge, JJ Thomson Avenue, CB3 OHE Cambridge, U.K.
}

\section{Supporting Information}

\begin{abstract}
Highly luminescent methylammonium lead bromide $\left(\mathrm{CH}_{3} \mathrm{NH}_{3} \mathrm{PbBr}_{3}\right)$ perovskite fibrous microstructure thin films have been fabricated using a perylene-3,4,9,10-tetracarboxylic dianhydridecontaining antisolvent-mediated single-step fabrication method. Confocal microscopy of these thin films reveals homogeneous emission over the entire area, in contrast to the localized emission from the thin films fabricated by conventional methods. The antisolvent treatment produces pinhole-free, mazelike morphology with high photoluminescence yield. These films were incorporated as the emissive layer in thin-film light emitting diodes (LEDs), made using a low-temperature meltable and processable alloy as cathode. These LEDs gave luminescence efficiency of up to $50 \mathrm{Cd} / \mathrm{m}^{2}$. The proposed LED structure highlights the prospects of using lowtemperature roll-to-roll processing for manufacturing perovskitebased solar cells and LEDs.
\end{abstract}

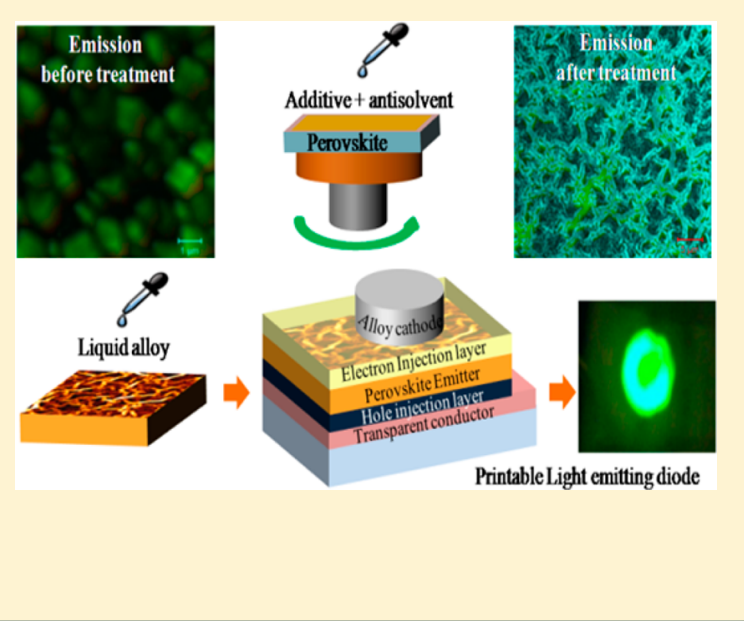

$\mathrm{O}$ rganic-inorganic hybrid perovskites (OIP) have been studied widely for their efficient light absorption and emission properties in their use to realize low-cost solar cells and light-emitting diodes (LEDs), respectively. ${ }^{1-5}$ The key advantages of hybrid perovskites lie in the superior charge generation and fast separation, ${ }^{6-8}$ while being easy to process at low temperatures. ${ }^{9-11}$ Hybrid perovskites show novel properties including high PL quantum yield (PLQY), ${ }^{12}$ tunable band gap, ${ }^{13,14}$ and structure and dimensionality tuning. ${ }^{15,16}$ Together with the capability to form various nanostructures, they provide a versatile set of materials for optoelectronic applications. ${ }^{3,12,17-20}$ Furthermore, perovskites with band gap in the visible range of the electromagnetic spectrum are also suitable for lighting and display applications. ${ }^{5}$ In this respect, $\mathrm{CH}_{3} \mathrm{NH}_{3} \mathrm{PbBr}_{3}$ perovskite has been studied in preference to the iodide variant, both because its emission lies in the visible spectrum (green) ${ }^{3}$ and also because it shows better stability ${ }^{21,22}$ and narrow emission width.

Morphological modification of OIP layers has been one of the key steps in improving the performance of OIP-based photovoltaics and LEDs. ${ }^{3,23,24}$ In general, a decrease in average grain size has been shown to enhance the resultant PL and electroluminescence (EL) and hence to improve the efficiencies of OIP-based LEDs. ${ }^{3}$ Tailored morphologies are specifically useful for anisotropic charge transport along preferred orientation, which are useful in field effect transistors. ${ }^{25,26}$
Aligned nanostructures have also been used for high photoconductive gain and array-based photodetectors. ${ }^{27-29}$ Crystal growth from solution phase has been of particular interest for obtaining hierarchical assemblies of single crystals and grains. ${ }^{30}$ According to the thermodynamic view, there are predominantly two different control routes (thermodynamic and kinetic) competing during the nucleation and crystal growth in faceted or three-dimensional orders. ${ }^{31}$ The crystal growth is driven by the second law of thermodynamics, and the resulting thin-film morphology adopts a configuration that achieves the lowest Gibbs free energy. Such processes may evolve over an extended period of time, overcoming the local energy minima before reaching the lowest-energy configuration. Alternatively, the crystal growth can be directed by the kinetic control route where rapid movement of solvents and grains controls the morphology. ${ }^{15}$ The final morphology tends to be a reflection of grains arrested in local energy minima which can be further modified by thermal annealing.

In this work, we demonstrate the role of kinetic growth control on morphological modifications of OIP thin films. We observe the growth of dendritic and mazelike structures upon

Received: October 25, 2016

Accepted: December 8, 2016

Published: December 8, 2016 
antisolvent treatment during the thin-film formation process. Significant differences in morphology are observed with the introduction of perylene-3,4,9,10-tetracarboxylic dianhydride (PTCDA) as additive in the antisolvent. Reduction in the grain size and morphological restructuring results in enhanced PLQY. To demonstrate the utility of these room-temperature processed, morphologically modified thin films in optoelectronic devices, we fabricated and tested LED devices. We have also used low-temperature (melting point, $\sim 58{ }^{\circ} \mathrm{C}$ ) eutectic alloy as a cathode to realize a vacuum-free, low-temperature, and all-solution-processed LED.

The perovskite layer with submicrometer crystals (SMCs) was fabricated using the additive-assisted microcrystal pinning process and demonstrates a unique morphological modification over conventional untreated perovskite thin films. One of the primary objectives of the additive-assisted submicrometer crystal pinning process is reduction of the grain size of perovskite crystals by inducing multiple seeding points, to achieve uniform, pinhole-free perovskite thin films. ${ }^{32}$ The grain size reduction is achieved by rapidly removing the better solvent (DMSO) from the film using an orthogonal solvent such as chloroform $\left(\mathrm{CHCl}_{3}\right)$, which results in rapid crystallization and reduced grain size. The representative atomic force microscopy (AFM) images of untreated, only solvent-treated microcrystals (S-SMCs), and A-SMC perovskite thin films (thickness, $\sim 300 \mathrm{~nm}$ ) are shown in Figure 1. The untreated film shows micrometer-sized $(\sim 5-10 \mu \mathrm{m})$, sparsely distributed grains with much less connectivity and large voids in between (Figure 1a). The S-SMC and A-SMC films show significant reduction in grain size $(\sim 100-400 \mathrm{~nm}$ determined using AFM line scan), with increased connectivity and considerably reduced pinholes (Figure $1 b, c$ ). Large-scale, mazelike structures are visible for S-SMC films (Figure 1c). Grain sizes are further reduced in A-SMC thin films, which results in increased PL yield. PTCDA additive-based SMC demonstrates a large variation in grain size $(\sim 100-300 \mathrm{~nm})$. The average size of OIP grains can be controlled by modifying the solubility of additives in the orthogonal solvent, which can be more effective in constricting the growth. From the classical theory of crystallization in the presence of small molecule additives, the additives attach to the surface of the crystalline grains through dangling bonds. Additives bonding with crystalline grains can restrict further crystallization, thereby controlling the average grain size. While being attached to crystalline grain on one side, the small molecules can interact with each other, resulting in a morphology favored by the stacking nature of the molecule. The fibrillar morphology observed in Figure 1c originates from the nature of perylenebased system to stack and form fibrillar networks. ${ }^{33}$ This morphology is unique to PTCDA or any perylene-based additive treatment. Changing PTCDA with $2,2^{\prime}, 2^{\prime \prime}-(1,3,5-$ benzinetriyl)-tris(1-phenyl-1-H-benzimidazole) (TPBi) (used by Cho et al. $^{3}$ ) results in a flat sheet type of morphology, as is shown in Figure S1.

The crystalline structure of OIP remains unchanged in the morphologically modified A-SMC thin films, as is evident from the X-ray diffraction (XRD) spectra shown in Figure 2a. Peaks visible at $2 \theta$ values of $14.9^{\circ}(100), 21.14^{\circ}(110), 30.11^{\circ}(200)$, $33.77^{\circ}(210), 37.12^{\circ}(211), 43.11^{\circ}(220)$, and $45.9^{\circ}(300)$ suggest a cubic symmetry prevalent in both the films. Lattice constant $(a)$ estimated from the XRD is $\sim 5.945 \AA$, which is in close agreement with previous reports. ${ }^{21,34}$ Absence of any additional peak signifies that the presence of PTCDA does not
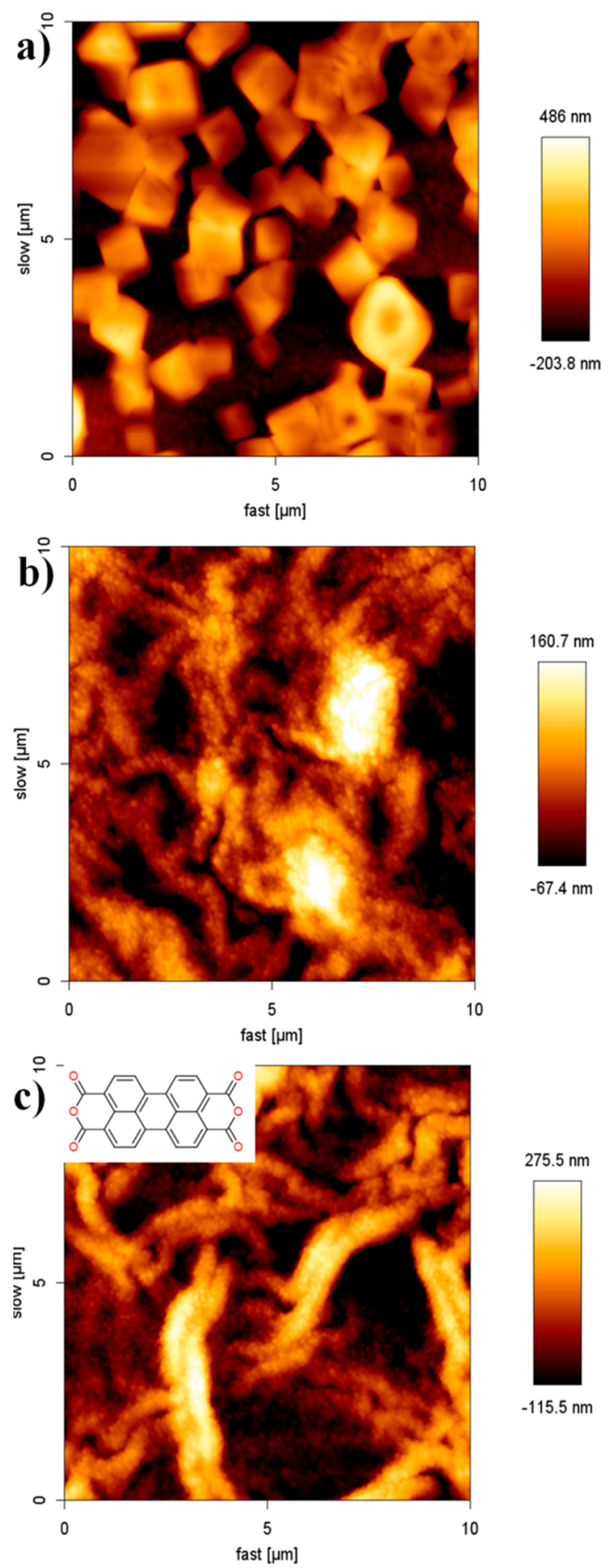

Figure 1. Submicrometer AFM morphology of OIP films. (a) The untreated film (control) shows micrometer-sized, sparsely distributed grains. (b) In the OIP film treated with solvent only, formation of dendritic structures can be seen. (c) OIP thin films treated with PTCDA/solvent show clear mazelike structures with submicrometer grains; the inset shows the chemical structure of PTCDA.

modify the crystal structure of OIP. The XRD peaks in untreated film are shifted to lower $\theta$ by $0.07^{\circ}$ compared to that of A-SMC thin films. A shift in the XRD peak can be correlated to stoichiometrically imbalanced OIP, which has smaller $a$. Such a shift can also be associated with the presence of compressive strain in a larger crystal, which can be relaxed 

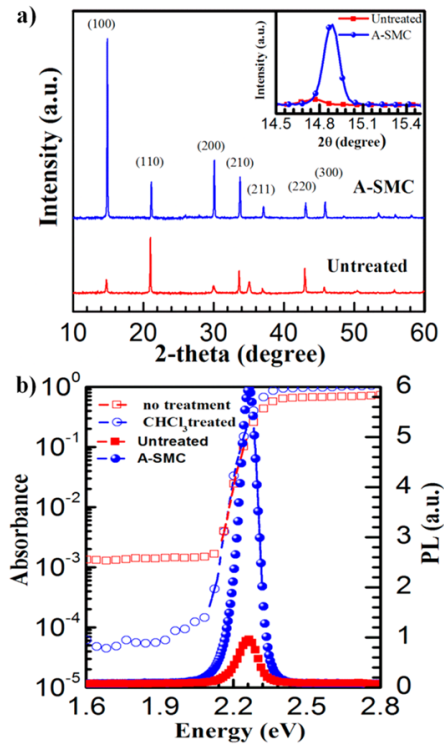

Figure 2. (a) Powder XRD spectra of untreated and A-SMC film. The inset shows a shift in the (100) peak for untreated (red, square) and A-SMC (blue, circle) films. (b) PDS absorbance for untreated (red, hollow square) and S-SMC films (blue, hollow circle) of OIP; PL emission from untreated (red, filled square) and A-SMC thin films (blue, filled circles) of OIP.

when the crystal size is reduced. Both films were fabricated from the same solution of lead bromide $\left(\mathrm{PbBr}_{2}\right)$ and methylammonium bromide (MABr) mixed in 1:1.05 molar ratio to ensure identical stoichiometry. Excess amount of $\mathrm{MABr}$ was used to ensure a complete conversion of $\mathrm{PbBr}_{2}$ to perovskite and avoid any metallic lead in the films, as was reported earlier. ${ }^{3}$ The boundary conditions for the formation of the grains can introduce a strain on the crystallites, resulting in a small shift in $a$.

To estimate the disorder in the semiconductor, we use photothermal deflection spectroscopy (PDS) technique, which can measure 4-5 orders of the dynamic range of absorption; this technique has been used extensively to investigate the quality of perovskite thin films. ${ }^{34,35}$ Absorption spectra measured using PDS show a considerable decrease (more than an order of magnitude) in sub-band gap absorption for solvent-modified films, as compared to that of untreated films (Figure 2b). A similar reduction was also observed for PTCDA additive-based SMC films. However, the low-lying band gap of PTCDA overlaps with the perovskite band-edge absorption, as shown in Figure S1. We expect the overall reduction in the defect density, based on the improved PL yields, and enhanced PL lifetimes. Significant reduction in nonradiative recombination rate is observed upon PTCDA additive-based A-SMC film, as is explained below. PTCDA signatures are present in the PDS absorbance of A-SMC films (figure S2), even after they are rinsed with chloroform. This suggests that the small molecules are embedded at the grain boundaries; similar observations have been reported with other OIP/small-molecule blend systems. ${ }^{36}$ Nevertheless, the observed decrease in PDS absorbance can be attributed to the decrease in the density of nonradiative defect states. This is also evident from PL measurements, where a large increase in the PL intensity (by a factor up to 6) is observed, as can be seen in Figure $2 \mathrm{~b}$. We attribute this increment to the decrease in nonradiative states and confinement of charge-pairs in small grains, which results in reduced delocalization and an increase in bulk and surface radiative recombination. ${ }^{3}$ The PLQY improves from $\sim 2 \%$ for untreated thin films to $25 \%$ for A-SMC thin films, which is consistent with the previous report. ${ }^{3}$ To further understand the photophysics of modified films, we have investigated the PL lifetimes for PTCDA-modified and unmodified films. The PL lifetime $(\tau)$ at low excitation fluence shows a considerable increase from $\sim 2 \mathrm{~ns}$ for untreated films to $\sim 36 \mathrm{~ns}$ for A-SMC films (Figure S3). From the PLQY and $\tau$ we calculate radiative $\left(k_{\mathrm{r}}\right)$ and nonradiative $\left(k_{\mathrm{nr}}\right)$ decay rates using PLQY $=k_{\mathrm{r}} /\left(k_{\mathrm{r}}+\right.$ $\left.k_{\mathrm{nr}}\right)$, and $\tau=1 /\left(k_{\mathrm{r}}+k_{\mathrm{nr}}\right)$. While the radiative rate increases slightly from $\sim 5 \times 10^{6} \mathrm{~s}^{-1}$ for untreated films to $\sim 7 \times 10^{6} \mathrm{~s}^{-1}$ for A-SMC films, the nonradiative component decreases by an order of magnitude, from $\sim 2.5 \times 10^{8} \mathrm{~s}^{-1}$ for untreated films to $\sim 2.3 \times 10^{7} \mathrm{~s}^{-1}$ for A-SMC films. This suggests a significant decrease in nonradiative states in A-SMC OIP films.

Localized PL was mapped, at submicrometer resolution, in order to probe the point-to-point variation in emission properties of morphologically modified thin films. The localized emission map is collected using a confocal fluorescence microscopy technique, which can provide a comprehensive image of emission from the modified thin films. A detailed spectral profile of emission has been created by sequentially gating the emission to different channels. Emission in untreated thin films is mostly localized on the cubelike grains, with a sharp decrease in emission outside the cube, as seen in the emission map in Figure 3a. The spectral profile across three
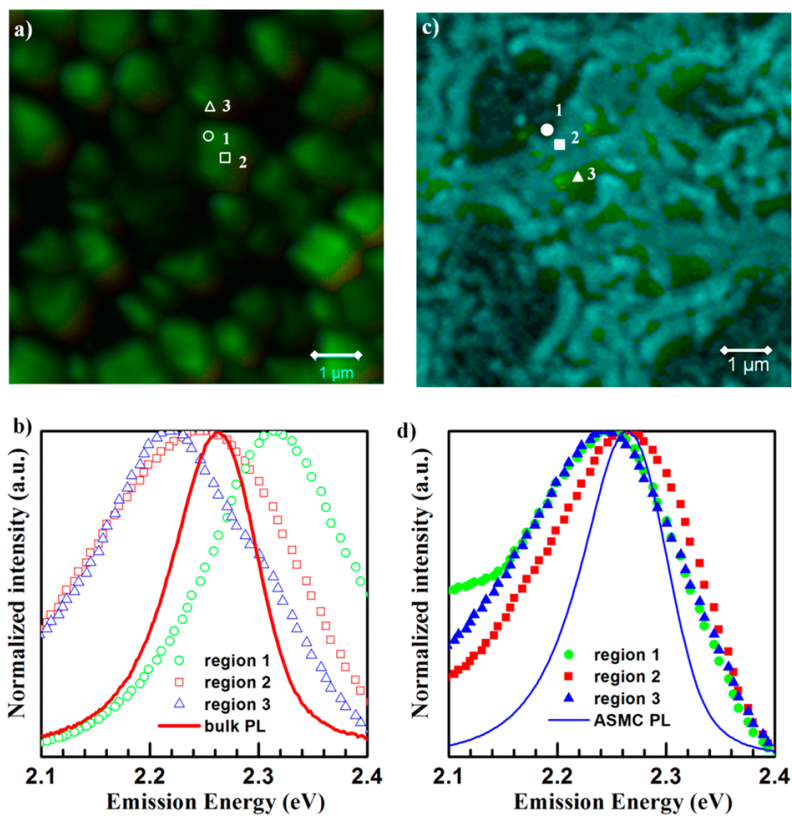

Figure 3. (a) Confocal spectral map of untreated thin films of OIP. (b) Emission spectra from three distinct regions (marked as 1, 2, and 3) of untreated OIP films. (c) Confocal spectral maps of ASMC thin films. (d) Emission spectra from three distinct (marked as 1,2 , and 3 ) regions across the fibrillar structure.

distinct regions of the cube (Figure $3 \mathrm{~b}$ ) shows a large shift in the emission peak across the grain (highlighted as regions 1,2 , and 3 in Figure 3a). In contrast, the emission is more homogeneously distributed for modified thin films, as can be seen in Figure 3c. The spectral distribution of the emission peak across the fibrillar structure is small compared to the variation in untreated films. The magnitude of emission closely 
follows the AFM topography, with fine details like the grain size and the submicrometer morphology becoming visible. The contrast in the bright and dark regions for the microstructures can be associated with the concentration of OIP grains present in the region. Higher variability in the emission peak for untreated films can arise from the large optical path length in the grains compared to the regions outside the grains where the OIP layer is much thinner. The emission spectral profile map suggests a red shift in emission in the regions between the grains compared to the bulk PL. Observed shifts can be associated with the internal reabsorption process or photon recycling effect in the dense regions where multiple reabsorptions can modify the emission profile on a macro scale. $^{37}$ The larger emission width for PL spectra measured through confocal techniques is due to the larger instrument response function. In contrast, the emission map of solventtreated thin films exhibit an inhomogeneous distribution of emission, and the emission profile does not seem to correlate with the observed microstructure (Figure S4). Microstructure ordering and emission tends to homogenize upon thermal annealing treatment (at $100{ }^{\circ} \mathrm{C}$ for $5 \mathrm{~min}$ ) of A-SMC thin film after spin coating (Figure S5). Observed improvements in morphologically modified thin films can be put to use by employing them in a working device like a photodetector or LED.

Large improvements have been observed in the photophysical and morphological properties of OIPs over conventional thin films made without any antisolvent processing. These modified films have been used as an emissive layer in low-temperature processed LEDs. One of the important technical advancements is the exploitation of a diverse family of low-temperature (melting temperature, $\sim 50-150{ }^{\circ} \mathrm{C}$ ) meltable alloy top contact which is well suited to the underlying solution-processed layers. The work function and interface charge transport properties are dependent on the composition and processing conditions, which significantly influence the device performance.

The vacuum-free cathode, fabricated using meltable alloy, exhibits superior charge injection properties compared to the conventional electrodes (which is reflected as higher injected current density in the LED device). These electrodes also offer better stability against moisture- and oxygen-related degradation by acting as an active encapsulant, as is evident from the long-term operational stability under atmospheric conditions. ${ }^{38}$ The meltable alloy is a quaternary eutectic mixture of indium, tin, lead, and bismuth. To probe the microstructure of this electrode in contact with the electron selective buffer layer, the top electrode of the LED was carefully peeled off and the surface in contact was examined. We observe micrometer-sized grains with low surface roughness, which matches the underlying film roughness (a scanning electron microscopy (SEM) image of the alloy electrode from the active area is shown in Figure S6a). The intimate conformal nature of the contact between the meltable alloy and the semiconductor film ensures an efficient charge injection, which leads to improvements in device performance over nontreated films. Elemental analysis of the alloy surface suggests the presence of components in their respective ratio, as shown in Figure S6b, resulting in an overall work function of $\sim 4.2 \mathrm{eV}$ (Figure S7).

LEDs fabricated using low-temperature-processed perovskite layer and vacuum-free alloy cathodes show stable EL under atmospheric conditions, as shown in Figure 4a. The A-SMC thin-film-based LEDs demonstrate higher external quantum
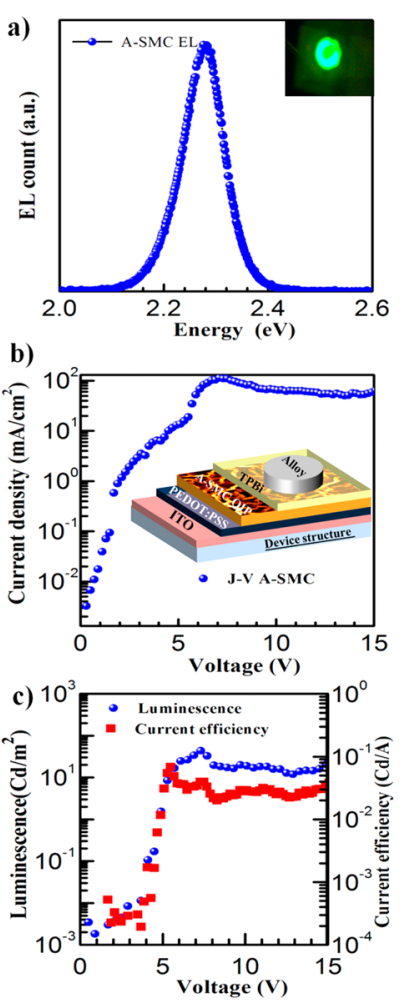

Figure 4. (a) EL emission from A-SMC thin films. The inset shows a working pixel of low-temperature processed LED. b) Current density vs voltage plot. The inset shows a schematic device structure. (c) Luminescence (blue, circle) and current efficiency (red, square) plot for PTCDA A-SMC thin-film-based LED.

efficiencies (EQEs) due to increased current efficiency and PLQY compared to that observed in the untreated thin film (where EL emission was not measurable). The charge-carrier mobility, measured using space charge limited current (SCLC) method for electron-only and hole-only devices, reveals a balanced charge transport $\left(\mu \approx 10^{-2} \mathrm{~cm}^{2} / \mathrm{V} \cdot \mathrm{s}\right.$ for both electron and hole), which is desirable for the recombination to occur in the OIP layer (Figure S8). A maximum current efficiency of $\sim 0.08 \mathrm{Cd} / \mathrm{A}$ was measured, the magnitude of which decreases to $\sim 35 \%$ of the maximum value (Figure $4 \mathrm{~b}$ ) at higher biases because of thermal burn in. ${ }^{39}$ The luminescence plot shows a steady increase in emission with voltage for A-SMC-based thinfilm LEDs, and an early saturation $(\sim 6 \mathrm{~V})$ is visible (Figure $4 \mathrm{c}$ ). A maximum luminescence of $\sim 50 \mathrm{Cd} / \mathrm{m}^{2}$ (Figure $4 \mathrm{c}$ ) and a peak EQE of $\sim 0.02 \%$ and IQE of $\sim 0.04 \%$ (Figure S9) has been realized. Above results show possible application of morphologically modified films in optoelectronic devices.

In conclusion, we demonstrate large-scale modification in microscopic morphology of OIP thin films using n-type small molecule-based additive mixed in orthogonal solvent, mediating kinetic control of the crystal growth process. Unique fibrillar and mazelike networks of OIP are observed in modified films, resulting from the kinetically driven submicrometer grains. Modified OIP films demonstrate a substantial decrease in disorder accompanied by a large increase in PL yield. Pinholefree, uniformly distributed morphology is evident from AFM while confocal studies show a homogeneous distribution of emission. Effects of photon recycling are visible in the localized PL map as the emission peak red-shifts with an increase in OIP thickness. The improvements observed in the OIP film are wellsuited for depositing soft alloy cathode from the melt state at 
low enough temperatures. Significant improvements in LED emission properties have been observed with modified films compared to that of untreated films. This process can also be utilized in scaled up, large area applications. The technique highlighted for the fabrication of fibrillar network will also be useful in realizing improvements in other OIP-based optoelectronic devices.

\section{EXPERIMENTAL SECTION}

Materials. MABr was procured from Dyesol, and $\mathrm{PbBr}_{2}$ was obtained from Alfa Asear (99.999\%). Small molecule 2,2',2' (1,3,5-benzinetriyl)-tris(1-phenyl-1- $H$-benzimidazole) (TPBi) was purchased from Sigma-Aldrich. Low-conductivity poly(3,4-ethylenedioxythiophene)-poly(styrenesulfonate)) (PEDOT:PSS) (clevios P VP AI 4083, resistivity 500-5000 $\Omega \mathrm{cm}$ ) was obtained from Heraeus. Low-temperature metal alloy electrodes were purchased from Roto Metals, United States.

Submicrometer Crystal Fabrication. ITO-coated glass substrates were cleaned by ultrasonication in acetone and isopropyl alcohol for $10 \mathrm{~min}$ each, followed by oxygen plasma for $10 \mathrm{~min}$. Low-conductivity PEDOT:PSS was spin-coated at $3000 \mathrm{rpm}$ under constant nitrogen flow, following which the thin films were dried in a nitrogen-filled glovebox at $180{ }^{\circ} \mathrm{C}$ for $10 \mathrm{~min}$. Further processing was performed in a nitrogen-filled glovebox for moisture and oxygen-free atmosphere. SMCs were formed by the additive-assisted submicrometer crystal pinning process; $0.2 \mathrm{wt} \%$ solution of PTCDA in $\mathrm{CHCl}_{3}$ was used as an additive. The perovskite layer was spin-coated at $3000 \mathrm{rpm}$, from a $40 \mathrm{wt}$ $\%$ solution of $\mathrm{PbBr}_{2}$ and $\mathrm{MABr}(1: 1.05$ molar ratio) in dimethyl sulfoxide (DMSO); additive was spin-coated on top of perovskite layer after $60 \mathrm{~s}$ of spinning, as reported previously. ${ }^{3}$ Pinning of crystals is governed by two competing processes, while the crystallites are arrested at submicrometer scale by removing the DMSO from the perovskite film; the orientation of crystallites is controlled by the small molecule additives. A thick layer of TPBi $(50-60 \mathrm{~nm})$ was spin-coated at $2000 \mathrm{rpm}$ from a $30 \mathrm{mg} / \mathrm{mL}$ solution in $\mathrm{CHCl}_{3}$ on top of $\mathrm{SMC}$. No additional annealing was required for SMC and TPBi layers.

Alloy Electrode Fabrication. The low-temperature $\left(58{ }^{\circ} \mathrm{C}\right)$ meltable alloy electrode used in this study has been tested for its charge injection and collection properties. From the previous studies, it is understood that the alloy performs on par with commonly used aluminum $(\mathrm{Al})$ as the cathode and demonstrates superior stability against oxidation as compared to $\mathrm{Al}$ electrodes. Liquid alloy is dispensed through a perforated polyethylene terephthalate (PET) mask, which defines the active area. Electrical measurements were performed after the alloy has cooled to room temperature.

Structural and Optical Characterizations. Thin films of untreated and PTCDA-modified OIP were spin-coated on quartz substrates. PL and quantum yield measurements were performed in an integrated PLQE setup. PL was collected using a fiber coupled silicon ( $\mathrm{Si}$ ) CCD-based spectrometer. A $405 \mathrm{~nm}$ laser light source was used as an excitation source. PL was measured using a power density of $100 \mathrm{~mW} / \mathrm{cm}^{2}$; PLQY was measured at $2.5 \mathrm{~W} / \mathrm{cm}^{2}$, and PL lifetimes were measured at $\sim 10^{16}$ photons $/ \mathrm{cm}^{3}$. XRD was carried out on SMC, and nontreated perovskite thin film was coated on ITO/ PEDOT:PSS substrates in a powder XRD setup. The $2 \theta$ values were varied from 10 to $90^{\circ}$; ITO peaks were subtracted from the SMC and bulk spectra.
Microscopy. AFM and confocal fluorescence imaging were performed on an integrated confocal (LSM 700) - AFM (nano wizard 3) from Carl Ziess and JPK instruments, Germany. AFM was performed in a noncontact tapping mode using a 300 $\mathrm{kHz}$ cantilever (force constant, $\sim 40 \mathrm{~N} / \mathrm{m}$ ). Confocal scanning were carried out using a $100 \times$ oil immersion objective; a 488 $\mathrm{nm}$ laser light source was used for excitation. Localized PL was recorded using a photomultiplier tube (PMT); for lambda scan, the emission was split into two channels using a variable dichroic mirror. SEM and energy-dispersive X-ray spectroscopy (EDAX) were performed in a Bruker instrument.

Electrical Characterization. LEDs fabricated using A-SMC as active layer and meltable alloy as back contact were characterized in a custom built LED characterization setup. Devices were sourced using Keithley 2400 source meter, and the LED emission was recorded using a reverse biased, calibrated $\mathrm{Si}$ detector and a combination of Keithley source meter and multimeter. EL spectrum was recorded using a fiber coupled Si CCD-based spectrometer (Ocean Optics).

\section{ASSOCIATED CONTENT}

\section{S Supporting Information}

The Supporting Information is available free of charge on the ACS Publications website at DOI: 10.1021/acsenergylett.6b00554.

Morphology of A-SMC with TPBi additive; PDS absorbance of A-SMC and PTCDA films; PL lifetime plots for untreated, S-SMC, and A-SMC films; AFM and confocal scans of S-SMC films and A-SMC post annealing films; SEM microstructure and EDAX studies of meltable alloy electrode surface; UPS plot for alloy work function; current density-voltage plots for SCLC mobility estimation; and LED EQE and IQE plot (PDF)

\section{AUTHOR INFORMATION}

\section{Corresponding Authors}

*E-mail: as2233@cam.ac.uk.

*E-mai: narayan@jncasr.ac.in.

ORCID ${ }^{\circledR}$

K.S. Narayan: 0000-0001-8550-6868

\section{Notes}

The authors declare no competing financial interest.

\section{ACKNOWLEDGMENTS}

We acknowledge UK-India Solar Energy Project; NewtonAPEX-II; Engineering and Physical Research Council, United Kingdom; and Department of Science and Technology, India for the funds. B.Z. acknowledges support from Cambridge Trust and China Scholarship Council. K.S.N. and P.K. acknowledge Dr. Subi Jacob George and Mr. Suman Kuila, NCU, JNCASR, Bangalore for helping with the TCSPC measurements.

\section{REFERENCES}

(1) Saliba, M.; Matsui, T.; Seo, J. Y.; Domanski, K.; Correa-Baena, J. P.; Nazeeruddin, M. K.; Zakeeruddin, S. M.; Tress, W.; Abate, A.; Hagfeldt, A.; et al. Cesium-Containing Triple Cation Perovskite Solar Cells: Improved Stability, Reproducibility and High Efficiency. Energy Environ. Sci. 2016, 9, 1989-1997.

(2) Yang, W. S.; Noh, J. H.; Jeon, N. J.; Kim, Y. C.; Ryu, S.; Seo, J.; Seok, S. I. High-Performance Photovoltaic Perovskite Layers 
Fabricated through Intramolecular Exchange. Science 2015, 348, 1234-1237.

(3) Cho, H.; Jeong, S.-H.; Park, M.-H.; Kim, Y.-H.; Wolf, C.; Lee, C.L.; Heo, J. H.; Sadhanala, A.; Myoung, N.; Yoo, S.; et al. Overcoming the Electroluminescence Efficiency Limitations of Perovskite LightEmitting Diodes. Science 2015, 350, 1222-1225.

(4) Tan, Z. K.; Moghaddam, R. S.; Lai, M. L.; Docampo, P.; Higler, R.; Deschler, F.; Price, M.; Sadhanala, A.; Pazos, L. M.; Credgington, D.; et al. Bright Light-Emitting Diodes Based on Organometal Halide Perovskite. Nat. Nanotechnol. 2014, 9, 687-692.

(5) Stranks, S. D.; Snaith, H. J. Metal-Halide Perovskites for Photovoltaic and Light-Emitting Devices. Nat. Nanotechnol. 2015, 10, 391-402.

(6) Ponseca, C. S.; Savenije, T. J.; Abdellah, M.; Zheng, K. B.; Yartsev, A.; Pascher, T.; Harlang, T.; Chabera, P.; Pullerits, T.; Stepanov, A.; et al. Organometal Halide Perovskite Solar Cell Materials Rationalized: Ultrafast Charge Generation, High and Microsecond-Long Balanced Mobilities, and Slow Recombination. J. Am. Chem. Soc. 2014, 136, 5189-5192.

(7) Stranks, S. D.; Eperon, G. E.; Grancini, G.; Menelaou, C.; Alcocer, M. J. P.; Leijtens, T.; Herz, L. M.; Petrozza, A.; Snaith, H. J. Electron-Hole Diffusion Lengths Exceeding 1 Micrometer in an Organometal Trihalide Perovskite Absorber. Science 2013, 342, 341344.

(8) Ponseca, C. S.; Tian, Y. X.; Sundstrom, V.; Scheblykin, I. G. Excited State and Charge-Carrier Dynamics in Perovskite Solar Cell Materials. Nanotechnology 2016, 27, 082001.

(9) Lee, M. M.; Teuscher, J.; Miyasaka, T.; Murakami, T. N.; Snaith, H. J. Efficient Hybrid Solar Cells Based on Meso-Superstructured Organometal Halide Perovskites. Science 2012, 338, 643-647.

(10) Kim, H. S.; Lee, C. R.; Im, J. H.; Lee, K. B.; Moehl, T.; Marchioro, A.; Moon, S. J.; Humphry-Baker, R.; Yum, J. H.; Moser, J. E.; et al. Lead Iodide Perovskite Sensitized All-Solid-State Submicron Thin Film Mesoscopic Solar Cell with Efficiency Exceeding 9\%. Sci. Rep. 2012, 2, 591.

(11) You, J. B.; Hong, Z. R.; Yang, Y.; Chen, Q.; Cai, M.; Song, T. B.; Chen, C. C.; Lu, S. R.; Liu, Y. S.; Zhou, H. P.; et al. Low-Temperature Solution-Processed Perovskite Solar Cells with High Efficiency and Flexibility. ACS Nano 2014, 8, 1674-1680.

(12) Deschler, F.; Price, M.; Pathak, S.; Klintberg, L. E.; Jarausch, D. D.; Higler, R.; Huttner, S.; Leijtens, T.; Stranks, S. D.; Snaith, H. J.; et al. High Photoluminescence Efficiency and Optically Pumped Lasing in Solution-Processed Mixed Halide Perovskite Semiconductors. J. Phys. Chem. Lett. 2014, 5, 1421-1426.

(13) Kumawat, N. K.; Dey, A.; Kumar, A.; Gopinathan, S. P.; Narasimhan, K. L.; Kabra, D. Band Gap Tuning of Ch3nh3pb(Br1Xclx)3 Hybrid Perovskite for Blue Electroluminescence. ACS Appl. Mater. Interfaces 2015, 7, 13119-13124.

(14) Noh, J. H.; Im, S. H.; Heo, J. H.; Mandal, T. N.; Seok, S. I. Chemical Management for Colorful, Efficient, and Stable InorganicOrganic Hybrid Nanostructured Solar Cells. Nano Lett. 2013, 13, 1764-1769.

(15) Zhu, P. C.; Gu, S.; Shen, X. P.; Xu, N.; Tan, Y. L.; Zhuang, S. D.; Deng, Y.; Lu, Z. D.; Wang, Z. L.; Zhu, J. Direct Conversion of Perovskite Thin Films into Nanowires with Kinetic Control for Flexible Optoelectronic Devices. Nano Lett. 2016, 16, 871-876.

(16) Im, J. H.; Luo, J. S.; Franckevicius, M.; Pellet, N.; Gao, P.; Moehl, T.; Zakeeruddin, S. M.; Nazeeruddin, M. K.; Gratzel, M.; Park, N. G. Nanowire Perovskite Solar Cell. Nano Lett. 2015, 15, 21202126.

(17) Fang, Y. J.; Dong, Q. F.; Shao, Y. C.; Yuan, Y. B.; Huang, J. S. Highly Narrowband Perovskite Single-Crystal Photodetectors Enabled by Surface-Charge Recombination. Nat. Photonics 2015, 9, 679-686.

(18) Chin, X. Y.; Cortecchia, D.; Yin, J.; Bruno, A.; Soci, C. Lead Iodide Perovskite Light-Emitting Field-Effect Transistor. Nat. Commun. 2015, 6, 7383.

(19) Luo, J. S.; Im, J. H.; Mayer, M. T.; Schreier, M.; Nazeeruddin, M. K.; Park, N. G.; Tilley, S. D.; Fan, H. J.; Gratzel, M. Water
Photolysis at $12.3 \%$ Efficiency Via Perovskite Photovoltaics and EarthAbundant Catalysts. Science 2014, 345, 1593-1596.

(20) Yakunin, S.; Sytnyk, M.; Kriegner, D.; Shrestha, S.; Richter, M.; Matt, G. J.; Azimi, H.; Brabec, C. J.; Stangl, J.; Kovalenko, M. V.; et al. Detection of X-Ray Photons by Solution-Processed Lead Halide Perovskites. Nat. Photonics 2015, 9, 444-449.

(21) Noh, J. H.; Im, S. H.; Heo, J. H.; Mandal, T. N.; Seok, S. I. Chemical Management for Colorful, Efficient, and Stable InorganicOrganic Hybrid Nanostructured Solar Cells. Nano Lett. 2013, 13, 1764-1769.

(22) Kim, Y. H.; Cho, H.; Heo, J. H.; Kim, T. S.; Myoung, N.; Lee, C. L.; Im, S. H.; Lee, T. W. Multicolored Organic/Inorganic Hybrid Perovskite Light-Emitting Diodes. Adv. Mater. 2015, 27, 1248-1254.

(23) Eperon, G. E.; Burlakov, V. M.; Docampo, P.; Goriely, A.; Snaith, H. J. Morphological Control for High Performance, SolutionProcessed Planar Heterojunction Perovskite Solar Cells. Adv. Funct. Mater. 2014, 24, 151-157.

(24) Li, G. R.; Tan, Z. K.; Di, D. W.; Lai, M. L.; Jiang, L.; Lim, J. H. W.; Friend, R. H.; Greenham, N. C. Efficient Light-Emitting Diodes Based on Nanocrystalline Perovskite in a Dielectric Polymer Matrix. Nano Lett. 2015, 15, 2640-2644.

(25) Lee, M. J.; Gupta, D.; Zhao, N.; Heeney, M.; McCulloch, I.; Sirringhaus, H. Anisotropy of Charge Transport in a Uniaxially Aligned and Chain-Extended, High-Mobility, Conjugated Polymer Semiconductor. Adv. Funct. Mater. 2011, 21, 932-940.

(26) Jimison, L. H.; Toney, M. F.; McCulloch, I.; Heeney, M.; Salleo, A. Charge-Transport Anisotropy Due to Grain Boundaries in Directionally Crystallized Thin Films of Regioregular Poly(3Hexylthiophene). Adv. Mater. 2009, 21, 1568-1572.

(27) Ai, N.; Zhou, Y.; Zheng, Y.; Chen, H.; Wang, J.; Pei, J.; Cao, Y. Achieving High Sensitivity in Single Organic Submicrometer Ribbon Based Photodetector through Surface Engineering. Org. Electron. 2013, 14, 1103-1108.

(28) Zhang, Y.; Wang, X.; Wu, Y.; Jie, J.; Zhang, X.; Xing, Y.; Wu, H.; Zou, B.; Zhang, X.; Zhang, X. Aligned Ultralong Nanowire Arrays and Their Application in Flexible Photodetector Devices. J. Mater. Chem. 2012, 22, 14357-14362.

(29) Zhang, X. J.; Jie, J. S.; Deng, W.; Shang, Q. X.; Wang, J. C.; Wang, H.; Chen, X. F.; Zhang, X. H. Alignment and Patterning of Ordered Small-Molecule Organic Semiconductor Micro-/Nanocrystals for Device Applications. Adv. Mater. 2016, 28, 2475-2503.

(30) Hooper, R. M.; McArdle, B. J.; Narang, R. S.; Sherwood, J. N. Chapter 10 - Crystallization from Solution at Low Temperatures. In Crystal Growth, 2nd ed.; Pamplin, B. R., Ed.; Pergamon: Oxford, 1980; Vol. 16, pp 395-420.

(31) Christian, P.; O'Brien, P. Thermodynamic and Kinetic Control of Crystal Growth in Cds Nanomaterials. J. Mater. Chem. 2008, 18, 1689-1693.

(32) Jeon, N. J.; Noh, J. H.; Kim, Y. C.; Yang, W. S.; Ryu, S.; Seok, S. I. Solvent Engineering for High-Performance Inorganic-Organic Hybrid Perovskite Solar Cells. Nat. Mater. 2014, 13, 897-903.

(33) Yan, K.; Chen, B. X.; Hu, H. W.; Chen, S.; Dong, B.; Gao, X.; Xiao, X. Y.; Zhou, J. B.; Zou, D. C. First Fiber-Shaped Non-Volatile Memory Device Based on Hybrid Organic-Inorganic Perovskite. Adv. Electron Mater. 2016, 2, 1600160.

(34) Sadhanala, A.; Deschler, F.; Thomas, T. H.; Dutton, S. E.; Goedel, K. C.; Hanusch, F. C.; Lai, M. L.; Steiner, U.; Bein, T.; Docampo, P.; et al. Preparation of Single-Phase Films of Ch3nh3pb$(\mathrm{I} 1-\mathrm{X}, \mathrm{Br}-\mathrm{X})(3)$ with Sharp Optical Band Edges. J. Phys. Chem. Lett. 2014, 5, 2501-2505.

(35) De Wolf, S.; Holovsky, J.; Moon, S. J.; Loper, P.; Niesen, B.; Ledinsky, M.; Haug, F. J.; Yum, J. H.; Ballif, C. Organometallic Halide Perovskites: Sharp Optical Absorption Edge and Its Relation to Photovoltaic Performance. J. Phys. Chem. Lett. 2014, 5, 1035-1039.

(36) Xu, J.; Buin, A.; Ip, A. H.; Li, W.; Voznyy, O.; Comin, R.; Yuan, M.; Jeon, S.; Ning, Z.; McDowell, J. J.; et al. Perovskite-Fullerene Hybrid Materials Suppress Hysteresis in Planar Diodes. Nat. Commun. 2015, 6, 7081. 
(37) Pazos-Outón, L. M.; Szumilo, M.; Lamboll, R.; Richter, J. M.; Crespo-Quesada, M.; Abdi-Jalebi, M.; Beeson, H. J.; Vrućinić, M.; Alsari, M.; Snaith, H. J.; et al. Photon Recycling in Lead Iodide Perovskite Solar Cells. Science 2016, 351, 1430-1433.

(38) Das, A. J.; Narayan, K. S. Retention of Power Conversion Efficiency - from Small Area to Large Area Polymer Solar Cells. Adv. Mater. 2013, 25, 2193-2199.

(39) Chang, M. H.; Das, D.; Varde, P. V.; Pecht, M. Light Emitting

Diodes Reliability Review. Microelectron. Reliab. 2012, 52, 762-782. 